\title{
Dynamic Modeling Method on Space Robot Dexterous Hand Based on Finite Element Theory
}

\author{
Lv Bohan ${ }^{1}$ \\ Department of Gas hydraulic servo control \\ The 18th Institute China Academy of Launch Vehicle \\ Technology \\ Beijing, China \\ E-mail: lbh19881230@126.com \\ Gu Jinshu ${ }^{3}$ \\ The 703th Institute China Academy of Launch Vehicle \\ Technology \\ Beijing, China
}

\author{
Liu Shan \\ Department of Gas hydraulic servo control \\ The 18th Institute China Academy of Launch Vehicle \\ Technology \\ Beijing, China
}

\author{
Zeng Guangshang ${ }^{4}$ \\ The 18th Institute China Academy of Launch Vehicle \\ Technology \\ Beijing, China
}

\begin{abstract}
Robot is a complex system consisting of multiple joints and connecting rods with complex coupling relationship between multiple inputs and multiple outputs, making it very difficult to establish the dynamic model of the robot. In order to realize the real-time control of the robot and improve the control precision, it is not enough to simply carry out dynamic simulation on the basis of ADAMS software. It is necessary to analyze the relationship between the driving force and torque of the system and the motion information from the robot mechanism. Based on the finite element theory, a simple and efficient modeling method of robot dynamics is presented in this paper. It has universal applicability for various complex robot systems, which can provide a theoretical basis for the high accuracy and real-time control of robot.
\end{abstract}

Keywords-robot, dexterous hand, finite element theory, dynamic modeling, motion control

\section{INTRODUCTION}

Robot is a complex system consisting of multiple joints and connecting rods with complex coupling relationship between multiple inputs and multiple outputs. The research on robot dynamics is to realize real-time control, then achieve the optimal control, the feedforward compensation and the improvement of the system performance. Therefore, it is very important to study the dynamics of the robot.

In order to accurately control the relative motion of each joint of the dexterous hand, only through the angular displacement information acquiring from the position sensors mounted on the joint is insufficient. Therefore, it is necessary to simplify the mechanical structure, analyze the degree of freedom of the manipulator system, and establish the dynamic model of the single finger movement. Then the relationship between the angular displacement, angular velocity and angular acceleration of the joint of the finger could be analyzed by means of mathematical analysis. Based on the kinematic analysis, the angular information of joint space is mapped to the end effector in Cartesian space, utilized as the theoretical basis of the motion control algorithm. Therefore the control precision of the robot could be improved.

\section{RESEARCH STATUS OF MECHANISM DYNAMIC ANALYSIS}

Regarding each part of dexterous fingers as rigid body, single finger dynamics modeling problem is essentially a multi rigid body system dynamics. The commonly method for dynamics modeling of mechanical system are Newton Euler method, Lagrange method, Kaine Houston equation and Robertson Witten Berg method.

Newton Euler method and Lagrange equation method have good practicability for the simple system with less components and freedom. With the increase of the number of components in the system, the connection condition and the constraint equations are becoming complex, then the establishment and solution of the equations of motion will be very difficult. With modern computing technology oriented, Robertson Wittenberg method has a great breakthrough in the regularity of the equations of motion and the application of computer friendly aspects. But the equation derivation process and means of expression are still complicated, sometimes it is not easy to use. In recent years, the Houston Kaine method is widely used to describe the topological structure of the system, using vector and transform matrix to describe the motion relationship between each part of the body with the dyadic representation of inertia. Therefore, based on Kaine equation, the kinematic equation is formed. Using the Kaine method, the appropriate generalized rate (i.e., pseudo velocity) is applied to describe the independent variables of the system. But the Kaine method is just a common method, and has not given a general formula. 
Since the birth of the finite element method, it has been widely used in the field of mechanical and civil engineering, and has become a powerful tool for the analysis of complex structures. However, the finite element method is not very common in the mechanism dynamics analysis. Because of the great difference between the rigid element and the structural analysis, the system cannot be used directly. In this paper, based on equivalent element integration theory and equivalent system, finite element method is applied to finite rigid component of dexterous robot hand complex mechanical system dynamic modeling.

\section{DYNAMIC MODELING METHOD BASED ON FINITE ELEMENT THEORY}

In the dynamic analysis of the dexterous hand based on the finite element method, the concept of equivalent element, equivalent system and equivalent force system are introduced. This equivalent is for the application of the finite element method on the assembly needed, and it is the equivalent of the dynamic and static characteristics of each member unit itself, thereby constituting the equivalent system. The purpose of the equivalent is to integrate, and not the traditional sense of the dynamic characteristics of the system to the equivalent of a certain component.

The mechanism consists of a number of components. Each component can be divided into one or several units. The movement of the unit depends on the unit's force and its own inertia. Two different units with different mass distribution may have the same inertia. In this regard, they will be named as the equivalent unit. The mass distribution between the equivalent units must meet certain conditions, so that the equivalent unit and the original unit inertia characteristics are the same.

Any one of the components of a quality uniform or not uniform can be equivalent to a lumped mass of component elements. The lumped mass dynamic model can be located at the centroid or ends of a member. See Figure 1.
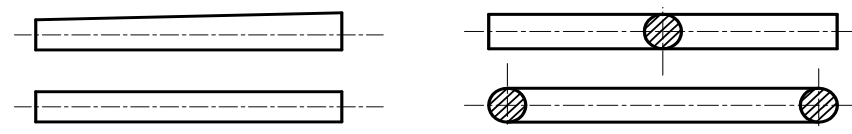

FIG. 1. COMPONENT ELEMENTAND AND EQUIVALENT ELEMENT

In engineering practice, the lumped mass unit is often used to replace the actual mass unit. In the dynamic analysis of multi rigid body system, the application of the equivalent lumped mass method in the element equivalent is special. In order to guarantee the equivalence between the equivalent lumped mass element and the original uniform or uneven mass element in the static and dynamic aspects, the inertia matrix of the unit should be guaranteed to be exactly the same as that of the original unit.

Using the principle of inertia equivalent, the equivalent element of concentrated mass distribution is obtained. The pseudo inertia matrix derived from the equivalent unit is exactly the same as the actual unit. However, lumped mass and inertia distribution bring great convenience for derivation of element motion equation and integrated assembly of system motion equations. For connecting rod mechanism, it is recommended that the mass concentration of the equivalent element at two ends of the rod is adopted, because the system is formed by the connection of the element node.

For rigid units, there are constraints

$$
\begin{aligned}
& \delta u_{i}=\sum_{a=1}^{n} \frac{\partial u_{i}}{\partial q_{a}} \delta q_{a}=\sum_{a=1}^{n} T_{i a} \delta q_{a} \\
& u_{i}=\sum_{b=1}^{n} \frac{\partial u_{i}}{\partial q_{b}} \dot{q}_{b}=\sum_{b=1}^{n} T_{i b} \cdot \\
& \ddot{u_{i}}=\sum_{b=1}^{n}\left(\frac{\partial u_{i}}{\partial q_{b}} \ddot{q_{b}}+\sum_{c=1}^{n} \frac{\partial^{2} u_{i}}{\partial q_{b} \partial q_{c}} \dot{q}_{b} \cdot \dot{q_{c}}\right)=\sum_{b=1}^{n} T_{i b} \ddot{q_{b}}+\sum_{b=1}^{n} \sum_{c=1}^{n} T_{i b c} \cdot \dot{q_{b}} \dot{q_{c}}
\end{aligned}
$$

The above constraints substituted into virtual displacement equilibrium equations

$$
\sum_{i, j, a} \delta q_{a} \cdot T_{i a} \cdot m_{i j}\left(T_{j b} \ddot{q_{b}}+T_{j b c}^{\prime} \cdot \dot{q_{b}} \dot{q}_{c}\right)=\sum_{i, a} \delta q_{a} \cdot T_{i a} \cdot F_{i}
$$

Substitute the constraints into virtual displacement equilibrium equations and obtain the equations of motion unit

$$
\sum_{b} m_{a b} \ddot{q_{b}}+\sum_{b, c} d_{a b c} \dot{q}_{b} \dot{q}_{c}=f_{a}
$$

Where

$$
m_{a b}=\sum_{i, j} T_{i a} m_{i j} T_{j b} \quad d_{a b c}=\sum_{i, j} T_{i a} m_{i j} T_{j b c}^{\prime} f_{a}=\sum_{i} T_{i a} f_{i}
$$

If the mechanism in the unit is considered as elastic unit with lumped inertia, from the unit to the system integration can fully use the finite element theory and methods, obtaining virtual displacement dynamic equilibrium equation of the system without the elastic stiffness.

$$
\sum_{i, j} u_{i}^{s} M_{i j}^{s} \ddot{u_{j}}=\sum_{i} \delta u_{i}^{s} F_{i}^{s}
$$

Among them, the relationship between the transition coordinates and generalized coordinates of the system is

$$
\begin{gathered}
\delta u_{i}=\sum_{a} T_{i a} q_{a} \quad \dot{u_{i}}=\sum_{b} T_{i b} \dot{q}_{b} \quad \ddot{u_{i}}=\sum_{b} T_{i b} \ddot{q_{b}}+\sum_{b, c} T_{i b c} \dot{q_{b}} \dot{q_{c}} \\
T_{i a}=\frac{\partial u_{i}}{\partial q_{a}} \quad T_{i b c}^{\prime}=\frac{\partial^{2} u_{i}}{\partial q_{b} \partial q_{c}}
\end{gathered}
$$

General expression of system dynamics are as follows 


$$
\sum_{b} M_{a b}^{s} \ddot{q_{b}}+\sum_{b, c} D_{a b c}^{s} \dot{q_{b}} \dot{q_{c}}=Q_{a}^{s}
$$

Or

$$
\sum_{a} T_{i a} M_{i}\left(\sum_{b} T_{i b} \ddot{q_{b}}+\sum_{b, c} T_{i b c}^{\prime} \cdot \dot{q_{b}} \dot{q}_{c}\right)=\sum_{a} T_{i a} F_{i}
$$

Where

$M_{a b}^{s}$ is two order mass matrix of the system in the generalized coordinates.

$D_{a b c}^{s}$ is three order mass matrix of the system in the generalized coordinates.

$Q_{a}^{s}$ is generalized force array of the system in the generalized coordinates.

$$
M_{a b}^{s}=\sum_{i, j} T_{i a} M_{i j} T_{j b} \quad D_{a b c}^{s}=\sum_{i, j} T_{i a} M_{i j} T_{j b c}^{j} Q_{a}^{s}=\sum_{i} T_{i a} F_{i}
$$

For complex mechanical systems, the establishment of the two order conversion matrix between the transition coordinates and the generalized coordinates is not easy. Jacobi matrix is often complex, and it is difficult to complete a full expression. Therefore, multiple integration method can be used to integrate, which can be considered as the transition coordinates and generalized coordinates of the equivalent system which is composed by the equivalent unit.

$$
\begin{gathered}
\frac{\partial u_{i}}{\partial q_{a}}=T_{i a}=\sum_{j} \sum_{k} \cdots \sum_{l} T_{i j}^{A} T_{j k}^{B} \cdots T_{l a}^{N} \\
{[T]=\left[T^{A}\right]\left[T^{B}\right] \cdots\left[T^{N}\right]} \\
T_{i b c} \frac{\partial^{2} u_{i}}{\partial q_{b} \partial q_{c}}=\sum_{j} \sum_{k} \cdots \sum_{l}\left(\frac{\partial T_{i a}^{A}}{\partial q_{c}}\right) T_{j k}^{B} \cdots T_{l b}^{N}+\cdots \bullet \\
+\sum_{j} \sum_{k} \cdots \sum_{l} T_{i j}^{A} T_{j k}^{B} \cdots\left(\frac{\partial T_{l b}^{N}}{\partial q_{c}}\right) \\
{\left[T^{\prime}\right]=\frac{\partial}{\partial q_{c}}\left(\left[T^{A}\right]\right)\left[T^{B}\right]\left[T^{C}\right] \cdots\left[T^{N}\right]+\cdots} \\
+\left[T^{A}\right]\left[T^{B}\right]\left[T^{C}\right] \cdots \frac{\partial}{\partial q_{c}}\left(\left[T^{N}\right]\right)
\end{gathered}
$$

By choosing appropriate intermediate coordinates, the intermediate conversion matrix $\left[T^{A}\right]\left[T^{B}\right]\left[T^{C}\right]$.. can be obtained. Therefore the complex conversion is decomposed into some simple intermediate conversion.

\section{CASE STUDY}

Therefore, this paper will establish the physical model of the single finger structure based on the finite element method, and establish the spatial coordinate system in each rotating joint. According to the degree of freedom of the system, the possible displacement of the joints is indicated. See figure 2.
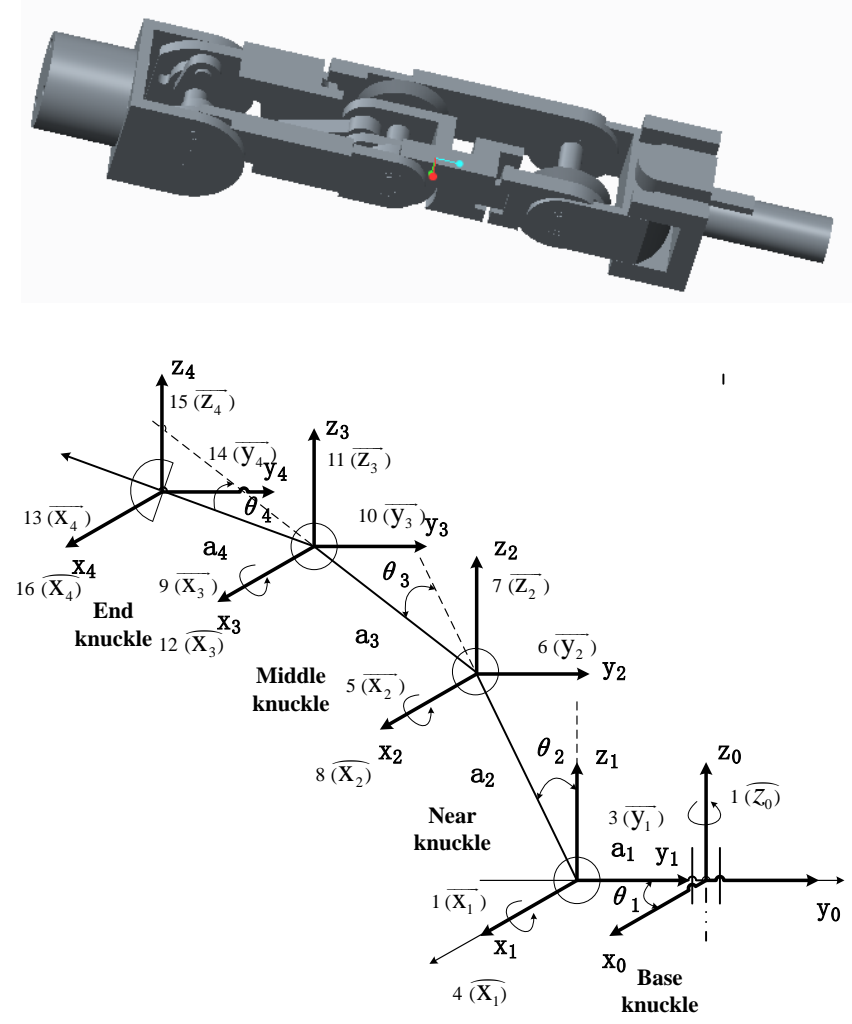

FIG. 2. PHYSICAL MODEL AND POSSIBLE DISPLACEMENT OF SINGLE FINGER STRUCTURE

Single finger system has 16 possible displacements. The system is divided into four units including base joint, near knuckle, middle knuckle and end knuckle. Each knuckle will centroid equivalent to the ends according to the space bar unit. See figure 3 .

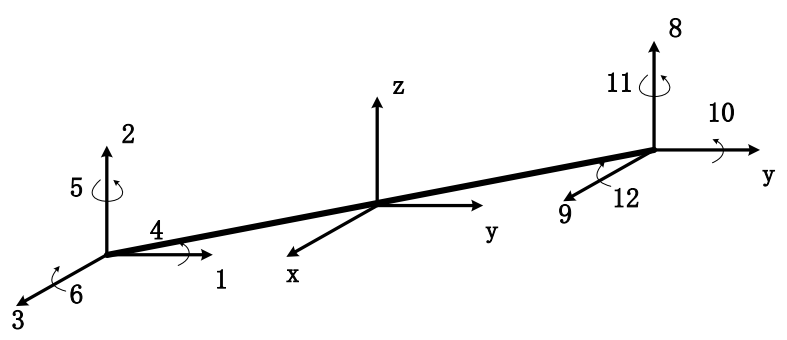

FIG. 3. A SPACE BAR UNIT WITH CENTROID EQUIVALENT TO TWO ENDS Its equivalent mass matrix is as follows. 


$$
m_{i i}=\frac{1}{2}\left[m, m, m, J_{x}, J_{y}, J_{z}, m, m, m, J_{x}, J_{y}, J_{z}\right]
$$

Where

$$
\begin{gathered}
m=A \rho l \\
J_{x}=\frac{1}{6} \rho l \cdot\left(I_{y}+I_{z}\right) ; J_{y}=\rho l \cdot\left(I_{y}-\frac{1}{6} A l^{2}\right) ; J_{z}=\rho l \cdot\left(I_{z}-\frac{1}{6} A l^{2}\right)
\end{gathered}
$$

Firstly, the correlation matrix $\left[N_{m}\right]$ of the unit node number and the system node number is listed.

$$
\left[N_{m}\right]=\left[\begin{array}{l}
0,0,0,0,0,1,2,3,0,4,0,0 \\
2,3,0,4,0,0,5,6,7,8,0,0 \\
5,6,7,8,0,0,9,10,11,12,0,0 \\
9,10,11,12,0,0,13,14,15,16,0,0
\end{array}\right]
$$

Then the equivalent mass matrix of the system $[M]$ is obtained.

$$
\begin{gathered}
{[M]=\operatorname{diag}\left[1_{6}, 1_{7}+2_{1}, 1_{8}+2_{2}, 1_{10}+2_{4}, 2_{7}+3_{1},\right.} \\
2_{8}+3_{2}, 2_{9}+3_{3}, 2_{10}+3_{4}, 3_{7}+4_{1}, \\
\left.3_{8}+4_{2}, 3_{9}+4_{3}, 3_{10}+4_{4}, 4_{7}, 4_{8}, 4_{9}, 4_{10}\right] \\
=\frac{1}{2} \operatorname{diag}\left[J_{12}, m_{1}+m_{2}, m_{1}+m_{2}, J_{1 x}+J_{2 x}, m_{2}+m_{3},\right. \\
\quad m_{2}+m_{3}, m_{2}+m_{3}, J_{2 x}+J_{3 x}, m_{3}+m_{4}, \\
\left.\quad m_{3}+m_{4}, m_{3}+m_{4}, J_{3 x}+J_{4 x}, m_{4}, m_{4}, m_{4}, J_{4 x}\right]
\end{gathered}
$$

Since the finger knuckles have different geometrical parameters, the system mass matrix is further expressed as

$$
\begin{aligned}
& {[M]=\operatorname{diag}\left[\frac{\rho a_{1} I_{1 z}}{2}-\frac{\rho A_{1} a_{1}^{3}}{12}, \frac{\rho\left(A_{1} a_{1}+A_{2} a_{2}\right)}{2},\right.} \\
& \frac{\rho\left(A_{1} a_{1}+A_{2} a_{2}\right)}{2}, \frac{\rho\left(I_{1 y} a_{1}+I_{1 z} a_{1}+I_{2 y} a_{2}+I_{2 z} a_{2}\right)}{12}, \\
& \frac{\rho\left(A_{2} a_{2}+A_{3} a_{3}\right)}{2}, \frac{\rho\left(A_{2} a_{2}+A_{3} a_{3}\right)}{2}, \frac{\rho\left(A_{2} a_{2}+A_{3} a_{3}\right)}{2}, \\
& \frac{\rho\left(I_{2 y} a_{2}+I_{2 z} a_{2}+I_{3 y} a_{3}+I_{3 z} a_{3}\right)}{12}, \frac{\rho\left(A_{3} a_{3}+A_{4} a_{4}\right)}{2}, \\
& \frac{\rho\left(A_{3} a_{3}+A_{4} a_{4}\right)}{2}, \frac{\rho\left(A_{3} a_{3}+A_{4} a_{4}\right)}{2}, \frac{\rho\left(I_{3 y} a_{3}+I_{3 z} a_{3}+I_{4 y} a_{4}+I_{4 z} a_{4}\right)}{12}, \\
& \left.\frac{\rho A_{4} a_{4}}{2}, \frac{\rho A_{4} a_{4}}{2}, \frac{\rho A_{4} a_{4}}{2}, \frac{\rho a_{4}\left(I_{4 y}+I_{4 z}\right)}{1}\right]
\end{aligned}
$$

According to stress, freedom and the relationship between coordinate of the single finger joints in three dimensional space, the generalized coordinates and generalized forces of the system are determined and dynamic model of the single finger of the dexterous hand is established.
The single finger system has 4 degrees of freedom, therefore, the rotation angle of each joint is used as the generalized coordinates of the system. Generalized coordinate matrix $q$ is as follows.

$$
q=\left[\theta_{1}, \theta_{2}, \theta_{3}, \theta_{4}\right]
$$

Where, $\theta_{3}$ and $\theta_{4}$ have a coupling relationship. The length of each knuckle is $a_{1}, a_{2}, a_{3}, a_{4}$ respectively.

Solve the possible displacement matrix of the system $[u]$

$$
[u]\left[\begin{array}{l}
u_{1}=\theta_{1} \\
u_{2}=a_{1} \cdot \cos \theta_{1} \\
u_{3}=a_{1} \cdot \sin \theta_{1} \\
u_{4}=\theta_{2} \\
u_{5}=\left(a_{2} \cdot \sin \theta_{2}+a_{1}\right) \cdot \cos \theta_{1} \\
u_{6}=\left(a_{2} \cdot \sin \theta_{2}+a_{1}\right) \cdot \sin \theta_{1} \\
u_{7}=a_{2} \cdot \cos \theta_{2} \\
u_{8}=\theta_{2}+\theta_{3} \\
u_{9}=\left[a_{3} \cdot \sin \left(\theta_{2}+\theta_{3}\right)+a_{2} \cdot \sin \theta_{2}+a_{1}\right] \cdot \cos \theta_{1} \\
u_{10}=\left[a_{3} \cdot \sin \left(\theta_{2}+\theta_{3}\right)+a_{2} \cdot \sin \theta_{2}+a_{1}\right] \cdot \sin \theta_{1} \\
u_{11}=a_{2} \cdot \cos \theta_{2}+a_{3} \cdot \cos \left(\theta_{2}+\theta_{3}\right) \\
u_{12}=\theta_{2}+\theta_{3}+\theta_{4} \\
u_{1}=\left[a_{4} \cdot \sin \left(\theta_{2}+\theta_{3}+\theta_{4}\right)+a_{3} \cdot \sin \left(\theta_{2}+\theta_{3}\right)+a_{2} \cdot \sin \theta_{2}+a_{1}\right] \cdot \cos \theta_{1} \\
u_{1}=\left[a_{4} \cdot \sin \left(\theta_{2}+\theta_{3}+\theta_{4}\right)+a_{3} \cdot \sin \left(\theta_{2}+\theta_{3}\right)+a_{2} \cdot \sin \theta_{2}+a_{1}\right] \cdot \sin \theta_{1} \\
u_{1}=a_{2} \cdot \cos \theta_{2}+a_{3} \cdot \cos \left(\theta_{2}+\theta_{3}\right)+a_{2} \cdot \cos \theta_{2}+a_{4} \cdot \cos \left(\theta_{2}+\theta_{3}+\theta_{4}\right) \\
u_{1}=\theta_{62}+\theta_{3}+\theta_{4}
\end{array}\right]
$$

In order to write simply, let $s_{1}=\sin \theta_{1}, \quad c_{1}=\cos \theta_{1}$, $S_{2}=\sin \theta_{2} \quad, \quad c_{2}=\cos \theta_{2}, \quad s_{3}=\sin \theta_{3}, \quad c_{3}=\cos \theta_{3}$, $s_{4}=\sin \theta_{4}, \quad c_{4}=\cos \theta_{4}$.

After simplification 
$[u]=$

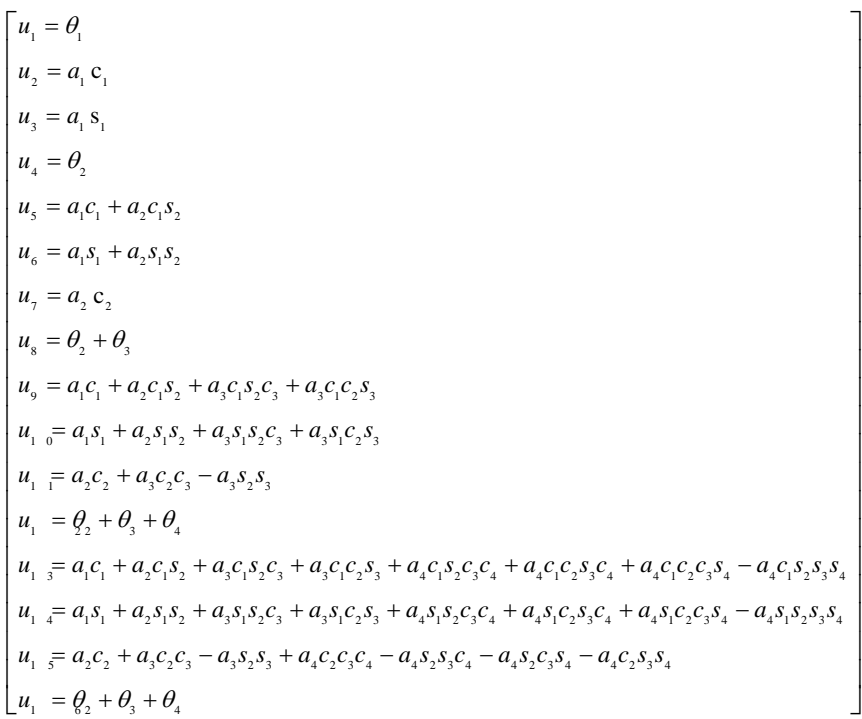

Respectively calculate $\left[T_{i a}\right]$ and $\left[T_{i b c}\right]$ matrix

$$
\left[T_{i a}\right]=\left[\frac{\partial u}{\partial \theta_{1}}, \frac{\partial u}{\partial \theta_{2}}, \frac{\partial u}{\partial \theta_{3}}, \frac{\partial u}{\partial \theta_{4}}\right]
$$

$$
\begin{aligned}
& {\left[T_{i b c}{ }^{\prime}\right]=} \\
& {\left[\frac{\partial^{2} u}{\partial \theta_{1}^{2}}, \frac{\partial^{2} u}{\partial \theta_{2} \partial \theta_{1}}, \frac{\partial^{2} u}{\partial \theta_{3} \partial \theta_{1}}, \frac{\partial^{2} u}{\partial \theta_{4} \partial \theta_{1}}, \frac{\partial^{2} u}{\partial \theta_{1} \partial \theta_{2}},\right.} \\
& \frac{\partial^{2} u}{\partial \theta_{2}^{2}}, \frac{\partial^{2} u}{\partial \theta_{3} \partial \theta_{2}}, \frac{\partial^{2} u}{\partial \theta_{4} \partial \theta_{2}}, \frac{\partial^{2} u}{\partial \theta_{1} \partial \theta_{3}}, \frac{\partial^{2} u}{\partial \theta_{2} \partial \theta_{3}}, \\
& \left.\frac{\partial^{2} u}{\partial \theta_{3}^{2}}, \frac{\partial^{2} u}{\partial \theta_{4} \partial \theta_{3}}, \frac{\partial^{2} u}{\partial \theta_{1} \partial \theta_{4}}, \frac{\partial^{2} u}{\partial \theta_{2} \partial \theta_{4}}, \frac{\partial^{2} u}{\partial \theta_{3} \partial \theta_{4}}, \frac{\partial^{2} u}{\partial \theta_{4}^{2}}\right]
\end{aligned}
$$

Suppose the driving torques of the 4 joints of the fingers are $M_{1}, M_{2}, M_{3}, M_{4}$, and generalized force matrix for the single finger system of the dexterous hand is $[F]$, then

$$
[F]=\left[M_{1}, 0,0, M_{2}, 0,0,0, M_{3}, 0,0,0, M_{4}, 0,0,0, M_{4}\right]^{T}
$$

Dynamic equation of single finger system for dexterous hand based on equivalent element integration method is as follows.

$$
\left[T_{i a}\right]^{T} \cdot[M] \cdot\left[T_{i a}\right] \cdot \ddot{q}+\left[T_{i a}\right]^{T} \cdot[M] \cdot\left[T_{i b c}\right] \cdot\left(q_{a} q_{b}\right)=\left[T_{i a}\right]^{T} \cdot[F]
$$

Among them, all the coefficient matrix of the dynamic equation has been solved.

$$
\vec{q}=\left[\ddot{\theta}_{1}, \ddot{\theta_{2}}, \ddot{\theta_{3}}, \ddot{\theta}_{4}\right]^{T} \text { is joint acceleration matrix. }
$$

$$
\begin{aligned}
\left(q_{a} q_{b}\right)= & {\left[\theta_{1}^{2}, \dot{\theta}_{1} \dot{\theta}_{2}, \dot{\theta}_{1} \dot{\theta}_{3}, \dot{\theta}_{1} \dot{\theta}_{4}, \dot{\theta}_{2} \dot{\theta}_{1}, \theta_{2}^{2}, \dot{\theta}_{2} \dot{\theta}_{3}, \dot{\theta}_{2} \dot{\theta}_{4},\right.} \\
& \left.\dot{\theta}_{3} \dot{\theta}_{1}, \dot{\theta}_{3} \dot{\theta}_{2}, \dot{\theta}_{3}^{2}, \dot{\theta}_{3} \dot{\theta}_{4}, \dot{\theta}_{4} \dot{\theta}_{1}, \dot{\theta}_{4} \dot{\theta}_{2}, \dot{\theta}_{4} \dot{\theta}_{3}, \dot{\theta}_{4}^{2}\right]^{T}
\end{aligned}
$$

velocity matrix.

\section{CONCLUSION}

The robot system is a complex, multi degree of freedom and highly coupled nonlinear dynamical system. Traditional dynamic modeling method such as Newton Euler method and Lagrange method are often constrained by large numbers of components and the complex constraint equations, leading to difficulties to establish the equation. Or the dynamic equation cannot be solved due to appearance of higher order derivatives. Besides it is not easy to use computers for analyzing. Based on the finite element method, a dynamic modeling method for complex robots is proposed, which only needs to solve the two order mass matrix, the three order mass matrix and the generalized force matrix. Theoretically, compared with Lagrange method and Newton Euler method in the field of robot dynamic modeling, its modeling process is simple and has high solving efficiency. Most significantly, this method solves the difficulties of complex system dynamic modeling.

\section{ACKNOWLEDGMENT}

We thank Liu Shan, Zeng Guangshang and Gu Jinshu for many insightful discussions. We are indebted to Wang Yanbo for providing experiment conditions and technical instrument. This work has been graciously funded under The 18th Institute China Academy of Launch Vehicle Technology.

\section{REFERENCES}

[1] Saeed B. Niku. Introduction to Robotics Analysis, Control, Applications, Second Edition. [M] 2014

[2] Jiansu Lai; Hong Xiang Lan. Identification of dynamic parameters in lagrange robot model, China Textile University, Systems, Proceedings of the 1988 IEEE International Conference on Pages: 90-93, 1988.

[3] Kanamori, M. Global stabilization of anti-windup PID position control for euler-lagrange systems with actuator saturation. Automation Science and Engineering (CASE), 2011 IEEE Conference on Pages 1-6, 2011.

[4] He Fuben, Liang Yande, Zhang Haohan, Pagello Enrico. Modeling, dynamics and control of an extended elastic actuator in musculoskeletal robot system, Advances in Intelligent Systems and Computing, Volume 194 AISC Issue VOL. 2, Pages: 671-681, 2013.

[5] Mei Shuai; Chenglong Fu; Chen, K. A Real-time Control Method for Humanoid Robot to Walk Stably on Uneven Ground, Robotics, Automation and Mechatronics, 2006 IEEE Conference on Pages: 1-6, Dec. 2006. 